\title{
Factors that differentially affect daytime and nighttime sleep in Drosophila melanogaster
}

\author{
Hiroshi Ishimoto ${ }^{1}$, Arianna Lark ${ }^{2}$ and Toshihiro Kitamoto ${ }^{1,2 *}$ \\ ' Department of Anesthesia, Carver College of Medicine, University of lowa, lowa City, IA, USA \\ 2 Interdisciplinary Graduate Program in Neuroscience, University of lowa, lowa City, IA, USA
}

\author{
Edited by: \\ Norio K. Ishida, National Institute of \\ Advanced Industrial Science and \\ Technology, Japan

\section{Reviewed by:} \\ Monica L. Andersen, Universidade \\ Federal de São Paulo, Brazil \\ Rakesh Bhattacharjee, University of \\ Chicago, USA \\ *Correspondence: \\ Toshihiro Kitamoto, Department of \\ Anesthesia, University of lowa, 1-316 \\ Bowen Science Building, 51 Newton \\ Road, lowa City, IA 52242, USA. \\ e-mail: toshi-kitamoto@uoiwa.edu
}

Rest in the fruit fly Drosophila melanogaster has key characteristics of mammalian sleep and is thus considered as a fly version of sleep. Drosophila sleep has been studied extensively, with the aim of gaining fundamental insights into the evolutionarily conserved functions of sleep as well as the mechanisms that regulate it. An interesting question that has not yet been addressed is whether fly sleep can be classified into distinct sleep types, each having particular biological roles - like rapid eye movement (REM) and non-REM sleep in birds and mammals. Typically, Drosophila sleep displays a bimodal pattern, consisting of distinct daytime and nighttime components. Notably, daytime and nighttime sleep differ with respect to several qualities, such as sleep-bout lengths and arousal thresholds. In this short review, we describe several genetic and environmental factors that differentially affect daytime and nighttime sleep, highlighting the observations suggesting the notion that these temporally distinct components of Drosophila sleep may have unique biological functions and be regulated by different homeostatic regulatory mechanisms.

Keywords: Drosophila melanogaster, sleep stage, daytime and nighttime sleep, sex peptide, ecdysone, angiotensinconverting enzyme-related gene

\section{INTRODUCTION}

Rest in the fruit fly Drosophila melanogaster is considered as a sleep-like state that shares many of the features of mammalian sleep (Hendricks et al., 2000; Shaw et al., 2000). Namely, Drosophila "sleep" is: controlled through both circadian and homeostatic mechanisms, (Huber et al., 2004), accompanied by specific alterations in gene expression (Cirelli et al., 2005), influenced by sex and age (Koh et al., 2006), and affected by pharmacological agents that are known to have effects on human sleep (Hendricks et al., 2000; Shaw et al., 2000). Even though many important aspects of mammalian sleep cannot be studied in fruit flies, the highly manipulable genome of Drosophila makes it an effective model organism for examining the fundamental functions of sleep, and the mechanisms that regulate it (Ho and Sehgal, 2005; Cirelli, 2009; Harbison et al., 2009).

In both mammals and birds, sleep is broadly classified into two distinct types: rapid eye movement (REM) sleep and nonREM (NREM) sleep. NREM sleep can be further divided into multiple discernible sleep stages, primarily based on their different electroencephalography (EEG) features (Rechtschaffen and Kales, 1968). These distinct sleep stages appear to be differentially regulated and to have unique biological functions (Siegel, 2005). Although it is not known if sleep in Drosophila is composed of multiple sleep stages, previous studies have demonstrated that clear qualitative differences exist between daytime and nighttime sleep (Hendricks et al., 2000; Shaw et al., 2000; Huber et al., 2004). An interesting inference from these findings is that Drosophila daytime and nighttime sleep may correspond to distinguishable physiological states that have specific biological functions and are regulated by overlapping but distinct mechanisms. As background to discussing this possibility, we briefly summarize the genetic and environmental factors known to differentially affect these two temporally distinct components of Drosophila sleep.

\section{GENERAL FEATURES OF DAYTIME AND NIGHTTIME SLEEP IN DROSOPHILA MELANOGASTER}

The fly's activity patterns can be easily and reliably monitored using the infrared beam-based Drosophila activity monitoring system (DAMS: Tri Kinetics, Waltham, MA, USA) or videobased methods (Zimmerman et al., 2008). Wild-type strains of D. melanogaster exhibit high levels of activity coinciding with light-on (dawn) and light-off (dusk) periods. Although substantial sexual dimorphism and strain-dependence are observed in Drosophila sleep architecture, wild-type flies spend time sleeping during both day and night. Using a standard definition of Drosophila sleep - a 5-min or longer interval with zero activity counts (no registered beam breaks) when monitored by DAMS (Hendricks et al., 2000; Shaw et al., 2000), a typical profile of Drosophila sleep displays a bimodal pattern, with peaks at noon and midnight and troughs at dawn and dusk. Drosophila sleep thus consists of two temporally distinct components: daytime sleep and nighttime sleep. These components differ in males and females. Male flies spend considerable time sleeping during both the day and night, with the amount of sleep during the day often comparable to that at night. Although mature mated females sleep as much as their male counterparts during the night, they exhibit sustained activity during day and sleep much less during this period (Huber et al., 2004).

Qualitative differences in daytime and nighttime sleep have been observed in both sexes. First, the average length of each 
sleep episode is significantly shorter during the day than the night. Second, arousal thresholds - sensory stimuli required to evoke behavioral responses - are higher for nighttime sleep than for daytime sleep (Huber et al., 2004). These observations suggest that daytime sleep is less intense, or shallower, than nighttime sleep. Whereas the occurrence of daytime and nighttime sleep is known to be controlled by circadian clocks, the mechanisms responsible for the qualitative differences between these sleep components remain largely uncharacterized.

One factor that is known to affect daytime and nighttime sleep differently is the aging process; its influence is particularly obvious in female flies. The amount of daytime sleep is highest in newly eclosed flies, and it declines over the course of 3 days, at which point a mature adult level is established. Nighttime sleep, in contrast, changes very little during the first 3 days of adult life. However, as the flies get older the amount of nighttime sleep starts to decline. By 33 days post-eclosion, the total amount of nighttime sleep is significantly below the third day level, whereas the total amount of daytime sleep does not decline further during this period (Shaw et al., 2000). In female flies, nighttime sleep decreases further as the end of life is approached ( $>60$ days), whereas daytime sleep increases. Notably, these extremely old flies spend considerable time sleeping at dawn and dusk (Koh et al., 2006). The overall effect of these changes is that sleep episodes in very old flies become distributed over the entire day, and the bimodal peaks for daytime and nighttime sleep become less obvious. Whether the qualitative differences between daytime and nighttime sleep are maintained in these aged flies remains to be determined.

A variety of genetic and environmental factors affect daytime or nighttime sleep preferentially. Some of these instances may simply reflect a floor or ceiling effect due to differences in baseline levels of sleep during the day or night. For example, if the basal sleep level at night is already maximal, leaving no room for sleep enhancement, a general sleep-promoting factor may appear to act only on daytime sleep (ceiling effect). Nevertheless, some factors indeed influence preferentially, if not exclusively, either daytime or nighttime sleep, as discussed below.

\section{MALE SEX PEPTIDE SELECTIVELY REDUCES DAYTIME SLEEP IN MATED FEMALE FLIES}

Sex peptide (SP) is a 36-amino-acid peptide that is synthesized by the male accessory gland and secreted into the seminal fluid. SP is known to cause various changes in the physiology and behavior of post-mated females, including a reduction in their sexual receptivity, an elevation in egg production, an increase in feeding rate, and an induction of antimicrobial peptides (Kubli, 2003). Whereas copulation does not have a strong influence on nighttime sleep in females, it leads to a significant $(\sim 60 \%)$ reduction in their daytime sleep (Isaac et al., 2010). This decrease in daytime sleep lasts at least 8 days after copulation. Isaac et al. (2010) have demonstrated that SP in post-mated females is responsible for promoting wakefulness and suppressing sleep during the day. Like other physiological and behavioral changes that are caused in mated females by the action of SP, the decrease in daytime sleep is assumed to have some benefits with respect to reproduction. For example, when mated females are more wakeful, they have more opportunities to obtain food, enabling them to produce larger numbers of eggs, and to find appropriate egg-laying sites, ensuring better survival of their progeny (Isaac et al., 2010). On the other hand, this behavioral change may also enhance risks imposed by unfavorable environmental conditions, such as low humidity during midday and afternoon. A better understanding of how SP reduces specifically daytime sleep is expected to provide important insight into a neural mechanism that controls the choice between the sleep vs. arousal state in the face of the physiological trade-off between the costs and benefits of each.

\section{LOSS OF ANGIOTENSIN-CONVERTING ENZYME-RELATED PEPTIDASE DISTURBS NIGHTTIME SLEEP}

Drosophila Acer encodes a member of the angiotensin-converting metallopeptidase family. Mammalian angiotensin-converting enzymes (ACE) generate angiotensin II, whose various roles include the regulation of blood pressure. In Drosophila, Acer mRNA cycles under the control of the Clock gene (McDonald and Rosbash, 2001). Two independent null mutations of Acer, which affect neither the rhythm of locomotor activity nor the amount of daytime sleep, result in a twofold to threefold increase in the total locomotor activity during the night. As a result, nighttime sleep is reduced in these Acer mutants (Carhan et al., 2011). The longest sleep-bout during the night is shortened by approximately $50 \%$ in the mutants, resulting in fragmented nighttime sleep. These phenotypes are not due to simple hyperactivity, because the locomotor activity of Acer mutants during wakeful episodes is not greater than that in control flies (Carhan et al., 2011). The effect of the Acer mutations can be reproduced by pharmacological disruption of angiotensin-converting enzyme-related (ACER) activity with fosinopril, the ACE inhibitor used in the treatment of hypertension and chronic heart failure. Compared to control flies, the duration of nighttime sleep bouts in flies fed fosinopril is reduced by $35 \%$. Daytime sleep-bout length is not significantly affected by this drug.

Drosophila Acer mRNA and protein are strongly expressed in the adult fat body (Carhan et al., 2011), which is physiologically analogous to liver and white adipose tissue in vertebrates, and is the organ primarily responsible for nutrient storage and metabolic control in Drosophila. Although the substrates of Drosophila ACER peptidase have not been identified, ACER may play a role similar to that of mammalian ACE in the processing of other peptide hormones, regulating energy, and metabolic homeostasis. Reduced nighttime sleep in Acer mutants could be explained by a disruption of metabolic control as a consequence of defects in ACER-dependent peptide hormones.

\section{LOSS-OF-FUNCTION MUTATIONS IN dArc LEAD TO INCREASES IN DAYTIME SLEEP AND DECREASES IN NIGHTTIME SLEEP}

In mammals, sleep and nutritional status are closely associated; food deprivation suppresses sleep (MacFadyen et al., 1973) and sleep deprivation promotes feeding (Rechtschaffen and Bergmann, 2002). In Drosophila, starvation similarly reduces sleep and induces higher locomotor activity (Keene et al., 2010). The activity-regulated cytoskeleton-associated protein gene (Arc) was initially identified in mammals as a neuronal immediate-early gene, showing rapid and robust upregulation with neuronal activity (Lyford et al., 1995). Consistent with those findings, $d A r c 1$, one of the three Drosophila Arc homologs, is upregulated in mutant 

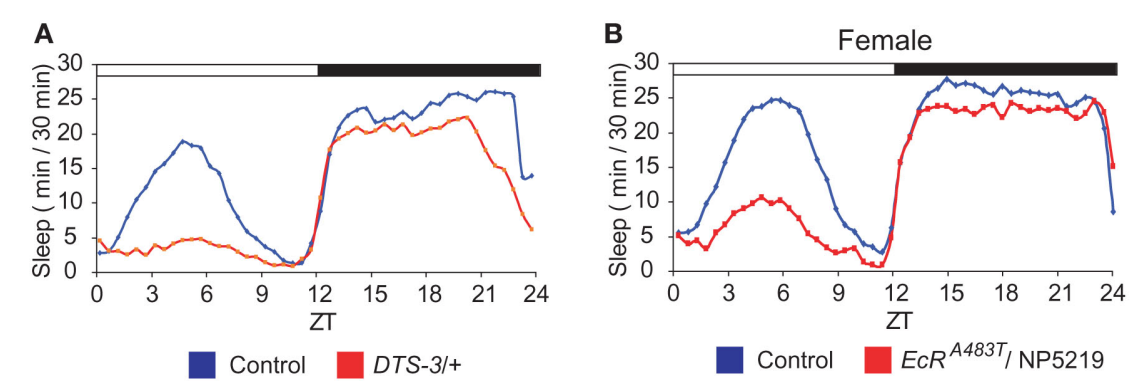

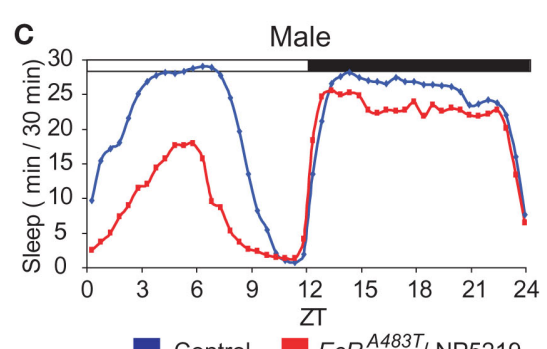

Control
FIGURE 1 | Down-regulation of ecdysone signaling as a consequence of mutations in DTS-3 and EcR results in significant suppression of

daytime sleep. Sleep patterns are shown for (A) a DTS-3 (mutant allele of molting defective, whose product is involved in ecdysone synthesis) heterozygous mutant, and (B,C) male and female $E_{C} R$ trans-heterozygous mutants ( $E C R^{\mathrm{A483T}} / \mathrm{NP5219}$ ), as well as the corresponding controls. Figures are adapted from Ishimoto and Kitamoto (2010). White and black horizontal bars represent the day (light-on) and night (light-off), respectively. flies that exhibit neuronal hyperexcitability (Guan et al., 2005). Loss-of-function mutations in $d A r c 1$ eliminate the normal hyperlocomotor response to starvation. $d A r c 1$ mutants survive without food longer than control flies, possibly because stored energy reserves last longer due to the absence of an increased activity upon starvation (Mattaliano et al., 2007). In addition to lacking the starvation-induced hyperlocomotion response, $d A r c$ mutants exhibit altered basal sleep patterns when the food supply is adequate. While the total amount of sleep in these flies is indistinguishable from that in controls, daytime and nighttime sleep are differentially affected; daytime sleep is increased and night time sleep is decreased (Mattaliano et al., 2007).

\section{REDUCED ECDYSONE SIGNALING SUPPRESSES DAYTIME SLEEP}

The molting steroid hormone 20-hydroxyecdysone (20E) has been shown to play a role in the regulation of sleep in adult flies (Ishimoto and Kitamoto, 2010). When ecdysone signaling is mitigated as a consequence of mutations in genes encoding either components of the ecdysone synthesis pathway or the nuclear ecdysone receptor $(E c R)$, the total amount of daytime sleep is drastically reduced. Notably, the same mutations affect nighttime sleep only slightly (Figure 1). Thus, daytime sleep seems to be more sensitive than nighttime sleep to a reduction in the strength of ecdysone signaling. The level of daytime sleep, but not nighttime sleep, is highest immediately after eclosion, and it decreases significantly during the first 3 days thereafter (Shaw et al., 2000). Since the level of $20 \mathrm{E}$ is highest in newly eclosed flies and declines as flies mature, the decrease in daytime sleep during early adulthood could be caused, at least in part, by an age-dependent reduction in the strength of ecdysone signaling.

\section{WARM AMBIENT TEMPERATURE HAS THE OPPOSITE EFFECT ON DAYTIME VS. NIGHTTIME SLEEP}

As observed in other animals, ambient temperature has significant effects on the wake-sleep states of Drosophila (Majercak et al., 1999; Chen et al., 2007). An active mechanism called "temperature compensation" ensures that the rhythms of locomotor activity are relatively constant over a broad range of physiological temperatures. However, endogenous circadian clocks can also adjust the daily distribution of wake and sleep periods in response to changes
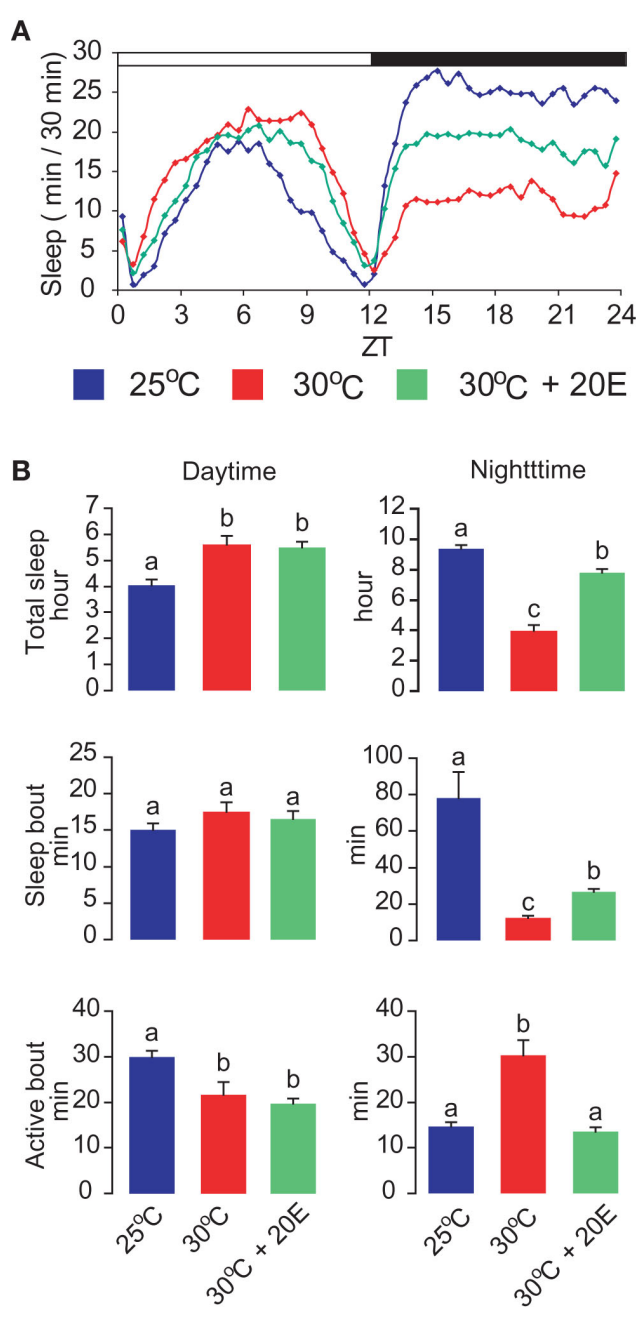

FIGURE 2 | Effects of warm ambient temperature and $20 \mathrm{E}$ administration on daytime and nighttime sleep. (A) Warm temperature enhances daytime sleep and suppresses nighttime sleep, and 20E antagonizes the effect of warm temperature on nighttime sleep. (B) Effects of warm temperature on total sleep time, sleep-bout length, and active-bout length, with or without feeding of $1 \mathrm{mM} 20 \mathrm{E}$. Letters above each bar indicate which groups differ significantly from one another (ANOVA, $P<0.05$ ). 
Table 1 | Factors differentially affecting daytime and nighttime sleep.

\begin{tabular}{|c|c|c|c|}
\hline Gene/treatment etc. & Related molecule/pathway & Effect & Reference \\
\hline Sex peptide & Peptide hormone & Daytime sleep $\downarrow$ & Isaac et al. (2010) \\
\hline Acer & Peptide hormone? & Nighttime sleep $\downarrow$ & Carhan et al. (2011) \\
\hline$E c R$ & Steroid hormone & Daytime sleep $\downarrow$ & Ishimoto and Kitamoto (2010) \\
\hline DTS-3 & Steroid hormone & Daytime sleep $\downarrow$ & Ishimoto and Kitamoto (2010) \\
\hline Hett PolyO & Neuronal disease & $\begin{array}{l}\text { Daytime sleep } \uparrow \\
\text { Fragmented nighttime sleep }\end{array}$ & Gonzales and Yin (2010) \\
\hline Fabps & Transport of lipophilic molecules & Daytime sleep $\uparrow$ & Gerstner et al. (2011) \\
\hline Aging & Oxidative stress & $\begin{array}{l}\text { Daytime sleep } \uparrow \text { (female) } \\
\text { Daytime sleep } \downarrow \text { (male) } \\
\text { Nighttime sleep } \downarrow \text { (female) }\end{array}$ & Koh et al. (2006) \\
\hline Infection Injury & Immune response & $\begin{array}{l}\text { Daytime sleep } \uparrow \\
\text { Daytime sleep } \uparrow\end{array}$ & Kuo et al. (2010) \\
\hline LNv hyperexcitation & Circadian rhythm & Nighttime sleep $\downarrow$ & Sheeba et al. (2008a) \\
\hline Warm temperature & Circadian rhythm & $\begin{array}{l}\text { Daytime sleep } \uparrow \\
\text { Nighttime sleep } \downarrow\end{array}$ & Chen et al. (2007) \\
\hline
\end{tabular}

in ambient temperature (Majercak et al., 1999). At warmer temperature (e.g., $30^{\circ} \mathrm{C}$ ), adult flies exhibit reduced locomotor activity and increased sleep levels during the day. Conversely, their nighttime activity is increased and their nighttime sleep is decreased (Figure 2A). When flies are shifted from cool to warm temperatures, the average length of the nighttime sleep-bout decreases and conversely the wake bout increases (Figure 2B). In contrast, the average length of daytime wake bouts decreases, and that of daytime sleep bouts remains unaltered. This temperature-induced modulation of sleep-wake periods is controlled primarily by a thermosensitive splicing event in the $3^{\prime}$ untranslated region of the clock gene period (per). Warm temperature attenuates the efficiency of per splicing, resulting in a delayed evening activity peak and enhancement of nocturnal activity, as well as suppression of nighttime sleep (Majercak et al., 1999). These opposite responses of daytime and nighttime sleep to warm temperature may have adaptive value. For instance, a reduction in foraging and other vital activities during the day at warm temperature can lower the risk of desiccation, whereas a reduction in nighttime sleep allows flies to engage in various activities at night under improved conditions.

The suppressive effect of warm ambient temperature on nighttime sleep is partially counteracted by oral administration of $20 \mathrm{E}$ (Figure 2A). Particularly noticeable is that the average wake-bout length, which is increased by approximately $100 \%$ at $30^{\circ} \mathrm{C}$ relative to $25^{\circ} \mathrm{C}$ levels, returns to the $25^{\circ} \mathrm{C}$ level when the flies are treated with 20E (Figure 2B). In contrast, the effects of warm ambient temperature on daytime sleep are not significantly influenced by 20E administration (Figure 2B). In Drosophila virilis, the levels of $20 \mathrm{E}$ are reported to increase after exposure to thermal stress $\left(38^{\circ} \mathrm{C}\right.$, $60 \mathrm{~min}$; Hirashima et al., 2000). Thus, the explanation for the failure of $20 \mathrm{E}$ administration to affect daytime sleep at warm temperatures may be that the long-term exposure to this environment and the resulting increase in $20 \mathrm{E}$ levels enhance daytime sleep to maximal levels.

The effect of warm temperature on sleep is also influenced by fatty-acid-binding proteins (Fabps), soluble proteins that serve as transporters for fatty acids, and other small lipophilic molecules.
The mRNA levels of the mouse brain-type Fabp (Fabp7) and its Drosophila homolog dFabp are diurnally regulated, with expression elevated during the main sleeping period (Gerstner et al., 2008, 2011). While overexpression of either Fabp7 or dFabp in Drosophila has little effect on nighttime sleep, it results in a significant reduction in baseline daytime sleep compared to that in control flies (Gerstner et al., 2011). Thus, the effect of Fabp overexpression on sleep is similar to that of reduced ecdysone signaling. This reduction in daytime sleep in the context of Fabp overexpression is not due to a general increase in locomotor activity, because waking activity (as measured by counts per waking minute) is decreased in this context. Rather, the overall reduction in sleep is due mainly to reduced sleep-bout length (Gerstner et al., 2011). At warm temperature, Fabp overexpression exaggerates the temperature-induced increase in daytime sleep. This is primarily due to an increase in the length of the maximum daytime sleep under these conditions (Gerstner et al., 2011).

\section{HYPEREXCITATION OF LARGE VENTRAL LATERAL CLOCK NEURONS LEADS TO A SPECIFIC REDUCTION IN NIGHTTIME SLEEP}

Internal circadian clocks, which control the daily rhythm of sleep-wake behavior, are composed of anatomically and functionally distinct groups of interconnected clock neurons in the brain. Among these, ventral lateral clock neurons are crucial for light entrainment of circadian behavior. The electrical activity of the large ventral lateral clock neurons (lLNvs) is regulated in circadian fashion, with their rate of spontaneous firing acutely increased in response to light (Sheeba et al., 2008b). Because the enhanced lLNvs activity driven by daytime light coincides with wakefulness and locomotor activity, ILNvs are thought to play a central role in modulating arousal and sleep. In fact, when ILNvs are electrically hyperexcited, the day-night pattern of the ILNvs peak firing rate is reversed, enhancing nocturnal locomotor activity and specifically suppressing nighttime sleep in a pigment-dispersing factor (PDF)-dependent manner (Sheeba et al., 2008a). 


\section{POSSIBLE FUNCTIONAL DIVERSITY OF DAYTIME AND NIGHTTIME SLEEP IN DROSOPHILA}

Although there are various theories about the potential roles of sleep, its exact functions continue to be an enigma. It is most likely that sleep fulfills multiple tasks necessary for an animal's survival and health, including replenishment of energy stores, removal of harmful by-products, and maintenance of neural plasticity. Different types of sleep and the different sleep stages observed in higher vertebrates seem to have rather selective roles in fulfilling certain functions of sleep. After sleep deprivation, for example, mammals tend to spend more time in NREM sleep, particularly slow-wave sleep (SWS), suggesting that SWS is the most important form in compensating for sleep loss. Although there is no clear evidence so far, for functional diversity of daytime and nighttime sleep in

\section{REFERENCES}

Carhan, A., Tang, K., Shirras, C. A., Shirras, A. D., and Isaac, R. E. (2011). Loss of Angiotensinconverting enzyme-related (ACER) peptidase disrupts night-time sleep in adult Drosophila melanogaster. J. Exp. Biol. 214, 680-686.

Chen, W. F., Low, K. H., Lim, C., and Edery, I. (2007). Thermosensitive splicing of a clock gene and seasonal adaptation. Cold Spring Harb. Symp. Quant. Biol. 72, 599-606.

Cirelli, C. (2009). The genetic and molecular regulation of sleep: from fruit flies to humans. Nat. Rev. Neurosci. 10, 549-560.

Cirelli, C., Lavaute, T. M., and Tononi, G. (2005). Sleep and wakefulness modulate gene expression in Drosophila. J. Neurochem. 94, 1411-1419.

Gerstner, J. R., Bremer, Q. Z., Vander Heyden, W. M., Lavaute, T. M., Yin, J. C., and Landry, C. F. (2008). Brain fatty acid binding protein (Fabp7) is diurnally regulated in astrocytes and hippocampal granule cell precursors in adult rodent brain. PLoS ONE 3, e1631. doi:10.1371/journal.pone.0001631

Gerstner, J. R., Vanderheyden, W. M., Shaw, P. J., Landry, C. F., and Yin, J. C. (2011). Fatty-acid binding proteins modulate sleep and enhance long-term memory consolidation in Drosophila. PLoS ONE 6, e15890. doi:10.1371/journal.pone.0015890

Gonzales, E., and Yin, J. (2010). Drosophila models of huntington's disease exhibit sleep abnormalities. PLoS Curr 2.

Guan, Z., Saraswati, S., Adolfsen, B., and Littleton, J. T. (2005). Genomewide transcriptional changes associated with enhanced activity in the Drosophila nervous system. Neuron 48, 91-107.

Harbison, S. T., Mackay, T. F., and Anholt, R. R. (2009). Understanding the neurogenetics of sleep: progress from Drosophila. Trends Genet. 25, 262-269.
Hendricks, J. C., Finn, S. M., Panckeri, K. A., Chavkin, J., Williams, J. A., Sehgal, A., and Pack, A. I. (2000). Rest in Drosophila is a sleep-like

Hirashima, A., Rauschenbach, I., and Sukhanova, M. (2000). Ecdysteroids in stress responsive and nonresponsive Drosophila virilis lines under stress conditions. Biosci. Biotechnol. Biochem. 64, 2657-2662.

Ho, K. S., and Sehgal, A. (2005). Drosophila melanogaster: an insect model for fundamental studies of sleep. Meth. Enzymol. 393, 772-793.

Huber, R., Hill, S. L., Holladay, C., Biesiadecki, M., Tononi, G., and sis in Drosophila melanogaster. Sleep 27, 628-639.

Isaac, R. E., Li, C., Leedale, A. E., and Shirras, A. D. (2010). Drosophila male sex peptide inhibits siesta sleep and promotes locomotor activity in the post-mated female. Proc. Biol. Sci. 277, 65-70.

Ishimoto, H., and Kitamoto, T. (2010). The steroid molting hormone ecdysone regulates sleep in adult Drosophila melanogaster. Genetics 185, 269-281.

Keene, A. C., Duboue, E. R., Mcdonald, D. M., Dus, M., Suh, G. S., Waddell, S., and Blau, J. (2010). Clock and cycle limit starvation-induced sleep loss in Drosophila. Curr. Biol. 20, 1209-1215.

Koh, K., Evans, J. M., Hendricks, J. C., and Sehgal, A. (2006). A Drosophila model for age-associated changes in sleep:wake cycles. Proc. Natl. Acad. Sci. U.S.A. 103, 13843-13847.

Kubli, E. (2003). Sex-peptides: seminal peptides of the Drosophila male. Cell. Mol. Life Sci. 60, 1689-1704.

Kuo, T. H., Pike, D. H., Beizaeipour, Z., and Williams, J. A. (2010). Sleep triggered by an immune response in Drosophila is regulated by the circadian clock and requires the NFkappaB Relish. BMC Neurosci. 11,17 . state. Neuron 25, 129-138. Cirelli, C. (2004). Sleep homeosta-

Drosophila, it would be worth investigating if these two temporally distinct sleep components also fulfill distinct functions. In addition to the genetic variants and treatments mentioned above, several factors affect daytime and nighttime sleep differentially in Drosophila (Table 1). While some of these may influence daytime or nighttime sleep by directly or indirectly modulating the circadian clock system, others may do so by affecting their homeostatic regulation. Studies of these factors are expected to provide important insights into specific roles of daytime and nighttime sleep in Drosophila, as well as into the mechanisms by which the observed qualitative differences arise. A better understanding of temporally distinct sleep components in Drosophila will likely shed light on the fundamental functions and regulatory mechanisms of sleep, which are likely conserved evolutionarily.

Lyford, G. L., Yamagata, K., Kaufmann, W. E., Barnes, C. A., Sanders, L. K., Copeland, N. G., Gilbert, D. J., Jenkins, N. A., Lanahan, A. A., and Worley, P. F. (1995). Arc, a growth factor and activity-regulated gene, encodes a novel cytoskeletonassociated protein that is enriched in neuronal dendrites. Neuron 14, 433-445.

MacFadyen, U. M., Oswald, I., and Lewis, S. A. (1973). Starvation and human slow-wave sleep. J. Appl. Physiol. 35, 391-394.

Majercak, J., Sidote, D., Hardin, P. E., and Edery, I. (1999). How a circadian clock adapts to seasonal decreases in temperature and day length. Neuron 24, 219-230.

Mattaliano, M. D., Montana, E. S., Parisky, K. M., Littleton, J. T., and Griffith, L. C. (2007). The Drosophila ARC homolog regulates behavioral responses to starvation. Mol. Cell. Neurosci. 36, 211-221.

McDonald, M. J., and Rosbash, M. (2001). Microarray analysis and organization of circadian gene expression in Drosophila. Cell 107, 567-578.

Rechtschaffen, A., and Bergmann, B. M. (2002). Sleep deprivation in the rat: an update of the 1989 paper. Sleep 25, 18-24.

Rechtschaffen, A., and Kales, A. (1968). A Manual of Standardized Terminology, Techniques and Scoring System for Sleep Stages of Human Subjects. Bethesda, MD: U.S. Dept. of Health, Education, and Welfare, Public Health Services-National Institutes of Health, National Institute of Neurological Diseases and Blindness, Neurological Information Network.

Shaw, P. J., Cirelli, C., Greenspan, R. J., and Tononi, G. (2000). Correlates of sleep and waking in Drosophila melanogaster. Science 287, 1834-1837.

Sheeba, V., Fogle, K. J., Kaneko, M., Rashid, S., Chou, Y. T.
Sharma, V. K., and Holmes, T. C. (2008a). Large ventral lateral neurons modulate arousal and sleep in Drosophila. Curr. Biol. 18, 1537-1545.

Sheeba, V., Gu, H., Sharma, V. K., O'dowd, D. K., and Holmes, T. C. (2008b). Circadian- and lightdependent regulation of resting membrane potential and spontaneous action potential firing of Drosophila circadian pacemaker neurons. J. Neurophysiol. 99, 976-988.

Siegel, J. M. (2005). Clues to the functions of mammalian sleep. Nature 437, 1264-1271.

Zimmerman, J. E., Raizen, D. M., Maycock, M. H., Maislin, G., and Pack, A. I. (2008). A video method to study Drosophila sleep. Sleep 31, 1587-1598.

Conflict of Interest Statement: The authors declare that the research was conducted in the absence of any commercial or financial relationships that could be construed as a potential conflict of interest.

Received: 21 October 2011; paper pending published: 21 November 2011; accepted: 09 February 2012; published online: 27 February 2012.

Citation: Ishimoto H, Lark A and Kitamoto $T$ (2012) Factors that differentially affect daytime and nighttime sleep in Drosophila melanogaster. Front. Neur. 3:24. doi: 10.3389/fneur.2012.00024 This article was submitted to Frontiers in Sleep and Chronobiology, a specialty of Frontiers in Neurology.

Copyright (c) 2012 Ishimoto, Lark and Kitamoto. This is an open-access article distributed under the terms of the Creative Commons Attribution Non Commercial License, which permits noncommercial use, distribution, and reproduction in other forums, provided the original authors and source are credited. 